\title{
A Comparison of Manifestations and Impact of Reassurance Seeking among Japanese Individuals with OCD and Depression
}

\author{
Osamu Kobori \\ Chiba University, Japan \\ Yoko Sawamiya \\ University of Tsukuba, Japan \\ Masaomi Iyo and Eiji Shimizu \\ Chiba University, Japan
}

\begin{abstract}
Background: One of the most common interpersonal reactions to threat and anxiety is to seek reassurance from a trusted person. The Reassurance Seeking Questionnaire (ReSQ) measures several key aspects of reassurance seeking behaviour, including frequency, trust of sources, intensity, carefulness, and the emotional consequences of reassurance seeking. Aims: The current study compares patterns and consequences of reassurance seeking in obsessivecompulsive disorder (OCD) and depression. Method: ReSQ scores were compared for three groups: 32 individuals with OCD, 17 individuals with depression, and 24 healthy comparison participants. Results: We found that individuals with OCD tended to seek reassurance more intensely and employ self-reassurance more frequently than individuals with depression or healthy participants, and that if reassurance was not provided, they tended to feel a greater urge to seek additional reassurance. Conclusions: This study is the first to quantitatively elucidate differences in reassurance seeking between OCD and depression.
\end{abstract}

Keywords: Obsessive-compulsive disorder, cognitive model, depression, reassurance seeking.

\section{Introduction}

\section{Reassurance seeking in obsessive-compulsive disorder and depression}

Excessive reassurance seeking is a common problem among both obsessive-compulsive and depressed populations, although it tends to differ in form and function between these populations. For example, individuals with OCD may ask others whether something is clean, whether they have done something properly, whether they are truly religious and so on. Other

Reprint requests to Osamu Kobori, Centre for Forensic Mental Health, Chiba University, 1-8-1, Inohana, Chuo-ku, Chiba, 2608670, Japan. E-mail: chelsea@ chiba-u.jp 
people are often involved in the processes of reassurance seeking and its provision, although sometimes individuals with OCD appear to reassure themselves or may consult impersonal sources, such as the Internet, to obtain reassurance (e.g. Kobori, Salkovskis, Read, Lounes and Wong, 2012; Kobori and Salkovskis, 2013). In contrast, reassurance seeking in depression has been defined as "the relatively stable tendency to excessively and persistently seek assurances from others that one is loveable and worthy, regardless of whether such assurance has already been provided" (Joiner, Metalsky, Katz and Beach, 1999, p. 270). Thus, reassurance seeking is likely to focus primarily on themes of low self-worth (e.g. "Do you think I'm boring?"), perceived threats of social loss or rejection (e.g. "Are you sure you're not mad at me?"), and/or the potential for failure due to personal incompetence (e.g. "Do you think I can handle this job/activity?").

Rachman's (2002) theory proposes that excessive reassurance seeking as well as compulsive checking in OCD can be construed as strategies aimed at exerting influence over negative outcomes and reducing one's perceived responsibility for such outcomes. However, few studies have investigated the functions of reassurance in OCD. Parrish and Radomsky (2006) asked healthy participants to perform a complex manual classification task (sorting pills) under conditions of high or low responsibility and threat. In the high-responsibility threat condition, participants were told that their results would be used to develop a safe and reliable system for sorting and distributing medications in a third-world country. Participants in the low-responsibility threat condition were told that the study sought to determine how quickly and accurately people could sort pills according to their colour and shape. Consistent with Rachman's (2002) theory, participants reported greater urges to check and seek reassurance under conditions of high (vs. low) responsibility and threat. This suggests that these two behaviours may be functionally equivalent and/or driven by similar processes.

Coyne's interactional model of depression (Coyne, 1976) proposes that reassurance seeking is used to determine whether others "truly" care about them, and to attempt to secure their relationships. Thus, reassurance-seeking episodes should cease once the depressed individual feels that they have gained sufficient evidence of caring from others. However, excessive reassurance seeking irritates others, and negative feedback from others (e.g. verbal criticism, displays of anger or frustration) increases the likelihood of social rejection and reinforces negative thoughts that one is not loveable or worthy; this in turn urges them to seek additional reassurance. Consistent with this model, excessive reassurance seeking has been shown to predict interpersonal rejection and severity of depression (see Starr and Davila, 2008, for a meta-analytic review).

In order to compare factors involved in the onset, maintenance, and termination of reassurance seeking and repeated checking in OCD and depression, Parrish and Radomsky (2010) employed a semi-structured interview with 15 non-depressed respondents with OCD, 15 clinically depressed individuals without OCD, and 20 healthy control participants. These researchers showed that while individuals with OCD reported seeking reassurance primarily concerning perceived general threats (e.g. fire, theft), the depressed group reported seeking reassurance primarily concerning perceived social threats (e.g. abandonment, loss of support, personal performance/competence). The primary motivations for both clinical groups to seek reassurance were to reduce anxious mood and to prevent harm. For individuals with depression, increased self-esteem and the prospect of receiving affection were additional motivations to seek reassurance. Regarding the termination of reassurance seeking, both anxiety reduction and interpersonal concerns were more likely to stop reassurance for both 
clinical groups, while healthy individuals and individuals with depression would stop seeking reassurance when they perceived a reduction of social threat.

\title{
Purpose of the present study
}

Several important issues remain to be investigated regarding reassurance seeking in OCD and depression. First, it is unknown from whom they seek reassurance. Kobori et al. (2012) suggested that individuals with OCD predominantly seek reassurance from someone close to them (e.g. partner, family members), but they also seek reassurance from books, websites, healthcare professionals, and themselves (i.e. self-reassurance). Thus, how frequently individuals with OCD and depression seek reassurance from such sources should be compared. Second, no study has quantitatively examined the function of reassurance seeking in OCD and depression. Although the perceived motivations of reassurance seeking have been identified, such as reductions of general/social threats (Parrish and Radomsky, 2010), the duration of such reductions and their feelings when not provided with reassurance remain undetermined.

Consequently, we employed the standardized Reassurance Seeking Questionnaire (ReSQ; Kobori and Salkovskis, 2013) in order to quantitatively compare reassurance seeking in OCD and depression. Comprising four scales and a separate section designed to assess reactions to obtaining reassurance, the ReSQ assesses a range of different aspects of reassurance seeking. Kobori and Salkovskis (2013) demonstrated that the ReSQ has good validity and reliability. Group comparisons between healthy participants and patients with OCD or panic disorder suggest that individuals with OCD seek reassurance more carefully (e.g. carefully listening to the person, becoming critical when the person does not seem to think seriously), and employ self-reassurance more frequently than individuals with panic disorder or healthy individuals. Moreover, using the "emotional changes" section of the questionnaire, Salkovskis and Kobori (2013) confirmed that reassurance improved individuals' emotions, but only temporarily, and that its effect decreased in the longterm. Moreover, if individuals failed to obtain reassurance, the resulting distress was greater for individuals with anxiety disorders than for healthy controls. This may help explain why others offer reassurance even when they know that reassurance works only temporarily.

In sum, the purpose of the present study is two-fold: (1) to quantitatively compare patterns of reassurance seeking in Japanese individuals with OCD and depression and healthy comparison participants; and (2) to quantitatively compare the consequences of obtaining reassurance on emotions in these groups. We hypothesized that individuals in clinical populations would demonstrate generally higher ReSQ scores than healthy comparisons, and that individuals with OCD would seek reassurance more frequently and intensely from sources other than people (e.g. books, websites, self-reassurance) than individuals with depression, but their emotional consequences of reassurance would be similar to those of individuals with depression.

\begin{abstract}
Method
Design

Individuals with OCD, individuals with depression, and healthy comparison participants took part in the questionnaire study. Participants were classified into three key criterion groups:
\end{abstract}


OCD, depression (DEP), and healthy comparison (HC). These groups were compared on several aspects of reassurance seeking using the ReSQ.

\section{Participants}

Initially, we recruited 32 individuals with OCD, 27 individuals with depression, and 29 healthy comparison participants. All participants identified their ethnicity and first language as Japanese. Individuals with OCD were recruited from among outpatients of Chiba University Hospital in Chiba, Japan. All had been diagnosed using the Structured Clinical Interview from the DSM-IV (SCID; First, Spitzer, Gibbon and Williams, 1995) by trained psychiatrists or psychologists. Individuals with depression were recruited through a charitable organization for depression and through their family members. Healthy participants were recruited through a charitable organization for individuals with depression and their families, and from among the administrative staff of Chiba University.

Although participants with depression had been diagnosed locally by their psychiatrists or physicians, the Structured Clinical Interview had not been used. Therefore we established exclusion criteria using standardized measures. Ten individuals with depression who scored less than 8 on the Beck Depression Inventory-II (BDI-II) or more than 60 on the Obsessive Compulsive Inventory(OCI) were excluded from the depression group. Similarly, five healthy comparison participants who scored more than 8 on the BDI-II or who scored more than 60 on the OCI were excluded from the healthy participant group. Thus we analyzed data from 32 individuals with OCD, 17 individuals with depression, and 24 healthy participants.

\section{Standardized measures}

Reassurance Seeking Questionnaire (ReSQ; Kobori and Salkovskis, 2013). The ReSQ has four scales and a separate section designed to assess emotional reactions.

Source. This section consists of 22 items to assess how frequently participants seek reassurance from different sources. Participants rate each item on a 6-point Likert scale ranging from 0 (never) to 5 (always). This section comprises five subscales: "involving other people in reassurance" (e.g. "I get other people to watch me when I do things which worry me"); "professionals" (e.g. "I seek reassurance from my family doctor"); "direct seeking from people" (e.g. "I seek reassurance from my partner"); "self-reassurance" (e.g. "I keep telling myself that there is nothing to worry about"); and "external references" (e.g. "I seek reassurance from websites").

Trust. This section concerns how much participants trust a range of information sources and consists of 16 items (e.g. "I trust reassurance from my partner"). Participants rate each item on a 6-point Likert scale ranging from 0 (not at all) to 5 (completely). The section comprises four subscales: "trust in people", "trust in health professionals", "trust in selfreassurance", and "trust in external references".

Intensity. This section asks how many times participants seek the same reassurance before stopping, and consists of 16 items (e.g. "I ask for reassurance from my partner"). Participants rate each item on a 5 -point Likert scale ranging from 0 (never) to 4 (many times). The section 
comprises four subscales: "direct seeking from people", "self-reassurance", "professionals" and "external references".

Carefulness. This section measures how careful participants become when they are seeking reassurance, and consists of 11 items. Participants rate each item on a 6-point Likert scale ranging from 0 (never) to 5 (always). The section comprises three subscales: "becoming critical" (e.g. "I show my frustration if the person gives only a vague reply"); "careful listening" (e.g. "I repeat what the person says so that they can confirm it"); and "caring for the person" (e.g. "I try not to ask too many times so I don't upset or annoy the person").

Emotional changes. This separate section assesses emotional reactions to obtaining vs. not obtaining reassurance, and asks how participants feel (reassured, anxious, guilty, or the urge to seek reassurance). Items are rated on a scale ranging from -5 (much less) to +5 (much more) for three different situations: (1) when the person they seek reassurance from does not answer or refuses to provide reassurance; (2) shortly after reassurance; and (3) 20 min or more after reassurance.

Development of the ReSQ-J. The Japanese version of the ReSQ (ReSQ-J) was developed using a standard back-translation procedure (Brislin, 1970, 1986), and its psychometric properties were tested with a sample of 129 Japanese undergraduates (Kobori and Sawamiya, 2012). The scales of the ReSQ-J had high internal consistency (Cronbach's alpha) and moderate to high test-retest reliability with a 2-week interval. Except for the trust scale, the other ReSQ-J scales had low to moderate correlations with the OCI total score and the BDI-II.

Obsessive-Compulsive Inventory (Foa, Kozak, Salkovskis, Coles and Amir, 1998). The OCI consists of 42 items and 7 subscales: washing, checking, doubting, ordering, obsessing (i.e. having obsessional thoughts), hoarding, and mental neutralizing. Each item is rated from 0 (has not troubled me at all) to 4 (troubled me extremely). We used the Japanese version of OCI developed by Ishikawa, Kobori and Shimizu (2013).

Beck Depression Inventory-II (Beck, Steer and Brown, 1996). The BDI-II is a wellvalidated, non-diagnostic 21-item self-report measure of depression severity in adults and adolescents. The inventory assesses cognitive, behavioural, and somatic features of depression over a one-week period. We used the Japanese version (BDI-II) developed by Kojima et al. (2002).

\section{Procedure}

In addition to standardized measures, participants were asked to indicate their marital status, employment status, and highest educational qualifications. They completed the questionnaire in their own time, mostly at home, and were given a 500-yen (approximately US\$ 5) gift voucher for their participation.

\section{Analysis}

The main analysis was a comparison of ReSQ scores for the three groups so that we could identify aspects of reassurance seeking specific to OCD, differing from depression, with the healthy participants providing a benchmark. We then analyzed emotional change (within and 
Table 1. Demographic status and general psychopathology measures

\begin{tabular}{lllll}
\hline & & OCD $(n=32)$ & DEP $(n=17)$ & HC $(n=24)$ \\
\hline Gender & Female:Male & $20: 12$ & $11: 6$ & $15: 9$ \\
Age & Mean $(S D)$ & $33.66(11.55)$ & $33.06(11.49)$ & $36.83(10.77)$ \\
Education & None or primary & $3 \%(1)$ & $6 \%(1)$ & $0 \%(0)$ \\
& Secondary or diploma & $56 \%(18)$ & $47 \%(8)$ & $13 \%(3)$ \\
& Degree or postgraduate & $41 \%(13)$ & $41 \%(7)$ & $79 \%(19)$ \\
Marital status & With partner (married, dating, & $37 \%(12)$ & $65 \%(11)$ & $67 \%(16)$ \\
& cohabiting) & & & \\
& Without partner (single, & $63 \%(20)$ & $35 \%(6)$ & $33 \%(8)$ \\
Occupation & divorced, widowed) & & & \\
& Employed or student & $37 \%(11)$ & $56 \%(10)$ & $83 \%(9)$ \\
OCI & Unemployed, not student & $63 \%(19)$ & $44 \%(7)$ & $17 \%(4)$ \\
BDI-II & $F(2,64)=40.511, p<.001$ & $74.86(33.29)^{\mathrm{a}}$ & $37.07(31.38)^{\mathrm{b}}$ & $13.79(10.62)^{\mathrm{c}}$ \\
& $F(2,64)=43.338, p<.001$ & $18.09(10.59)^{\mathrm{b}}$ & $28.18(10.63)^{\mathrm{a}}$ & $2.92(2.685)^{\mathrm{c}}$ \\
\hline
\end{tabular}

Notes: $\mathrm{OCD}=$ Obsessive-Compulsive Disorder, DEP $=$ Depression, $\mathrm{HC}=$ Healthy Comparison, OCI $=$ Obsessive-Compulsive Inventory, BDI-II $=$ Beck Depression Inventory-II. The different superscript letters indicate a significant difference between groups, based on a Bonferroni multiple comparison test $(p<.05)$.

between groups) to demonstrate how each group of participants reported reacting when they were not able to obtain reassurance, and when they received reassurance in the short or long term.

Prior to conducting the main analysis, we examined group differences in demographics and psychopathology. We found no differences between groups in age, gender, or occupational status. Marital status differed somewhat, $\chi^{2}(2)=5.82, p=.054$, in that fewer individuals with OCD were married. Healthy individuals had higher levels of education than participants in the clinical groups, $\chi^{2}(4)=12.79, p=.010$ (Table 1 ).

\section{Results}

\section{Group comparisons for ReSQ scales and subscales}

The analysis first compared the total scores on the four domains of the ReSQ among the three groups; the subscales of each domain were then examined separately. The three groups differed on all scales of the ReSQ. A $3 \times 4$ mixed-model multivariate analysis of variance (MANOVA) was conducted to compare the three groups (OCD, DEP, and HC) across the four scales of the ReSQ (source, trust, intensity, and carefulness). The MANOVA revealed significant main group effects $F(8,108)=2.13, p=.038, p \eta^{2}=.137$. Follow-up ANOVAs revealed significant main effects of group on all of the scales, and post hoc multiple comparisons using Tukey's HSD test revealed that the OCD group had higher scores on the intensity subscale relative to the HC group (Table 2).

The three groups differed on the source subscale of the ReSQ. A MANOVA revealed significant main effects of group (between factors), $F(10,118)=4.82, p<.001, p \eta^{2}=$ .234. Follow-up ANOVAs revealed significant main effects of group on the professionals, 
Table 2. Group differences on ReSQ scales and subscales

\begin{tabular}{|c|c|c|c|c|c|c|}
\hline \multirow{2}{*}{$\frac{\text { Variable }}{\text { Source }}$} & \multicolumn{2}{|c|}{$\begin{array}{c}\text { OCD } \\
(n=32) \\
\text { Mean }(S D)\end{array}$} & \multicolumn{2}{|c|}{$\begin{array}{l}\text { Depression } \\
(n=17) \\
\text { Mean }(S D)\end{array}$} & \multicolumn{2}{|c|}{$\begin{array}{l}\text { Healthy comparisons } \\
(n=24) \\
\text { Mean }(S D)\end{array}$} \\
\hline & 1.420 & $(0.759)$ & 1.540 & $(0.447)$ & 1.129 & $(0.427)$ \\
\hline Trust & 2.160 & $(0.677)$ & 2.244 & $(0.778)$ & 1.734 & $(0.709)$ \\
\hline Intensity & $1.530^{\mathrm{a}}$ & $(0.523)$ & 1.310 & $(0.631)$ & $0.961^{\mathrm{b}}$ & $(0.460)$ \\
\hline Carefulness & 2.597 & $(0.973)$ & 2.667 & $(0.662)$ & 2.044 & $(0.690)$ \\
\hline \multicolumn{7}{|l|}{ Source subscales: } \\
\hline $\begin{array}{l}\text { Involving other people in } \\
\text { reassurance }\end{array}$ & 1.679 & $(1.095)$ & 1.708 & $(0.682)$ & 1.283 & $(0.576)$ \\
\hline Professionals & 0.846 & $(0.918)$ & $1.175^{\mathrm{a}}$ & $(0.762)$ & $0.530^{\mathrm{b}}$ & $(0.466)$ \\
\hline Direct seeking from people & 1.471 & $(1.165)$ & 1.297 & $(0.678)$ & 1.033 & $(0.545)$ \\
\hline Self-reassurance & $2.365^{\mathrm{a}}$ & $(0.914)$ & $1.516^{\mathrm{b}}$ & $(0.750)$ & $1.326^{\mathrm{b}}$ & $(0.680)$ \\
\hline External reference & $0.846^{\mathrm{b}}$ & $(0.822)$ & $1.719^{\mathrm{a}}$ & (1.154) & 1.261 & $(0.987)$ \\
\hline \multicolumn{7}{|l|}{ Trust subscales: } \\
\hline Trust in people & 1.823 & $(0.877)$ & 2.000 & $(0.770)$ & 1.704 & $(0.757)$ \\
\hline Trust in health professionals & 2.025 & $(1.399)$ & $2.578^{\mathrm{a}}$ & $(1.050)$ & $1.250^{\mathrm{b}}$ & (1.109) \\
\hline Trust in self-reassurance & 2.432 & $(1.053)$ & 2.133 & $(1.075)$ & 1.847 & (1.077) \\
\hline Trust in external references & 2.093 & $(0.951)$ & 2.267 & $(0.904)$ & 1.729 & $(0.847)$ \\
\hline \multicolumn{7}{|l|}{ Intensity subscales: } \\
\hline Direct seeking from people & $1.700^{\mathrm{a}}$ & $(0.730)$ & $1.022^{\mathrm{b}}$ & $(0.704)$ & $0.928^{b}$ & $(0.479)$ \\
\hline Self-reassurance & $2.507^{\mathrm{a}}$ & $(0.991)$ & 1.778 & $(1.180)$ & $1.261^{\mathrm{b}}$ & $(0.674)$ \\
\hline Professionals & 0.632 & $(0.685)$ & 0.907 & $(0.580)$ & 0.487 & $(0.455)$ \\
\hline External references & 1.260 & $(0.926)$ & 1.533 & $(1.043)$ & 1.000 & $(0.783)$ \\
\hline \multicolumn{7}{|l|}{ Carefulness subscales: } \\
\hline Becoming critical & $2.643^{\mathrm{a}}$ & $(1.218)$ & 2.594 & $(0.944)$ & $1.865^{\mathrm{b}}$ & $(0.972)$ \\
\hline Careful listening & 2.527 & $(1.274)$ & $2.797^{\mathrm{a}}$ & $(0.900)$ & $1.969^{\mathrm{b}}$ & $(0.760)$ \\
\hline Caring for the person & 2.560 & $(0.837)$ & 2.625 & $(0.934)$ & 2.083 & $(0.929)$ \\
\hline
\end{tabular}

Notes: ReSQ = Reassurance Seeking Questionnaire, OCD = Obsessive-Compulsive Disorder. The different superscript letters indicate a significant difference between groups based on a Bonferroni multiple comparison test $(p<.05)$. Source and its subscales were rated from 0 (never) to 5 (always); Trust and its subscales were rated from 0 (not at all) to 5 (completely); Intensity and its subscales were rated from 0 (never) to 4 (many times); Intensity and its subscales were rated from 0 (never) to 5 (always).

self-reassurance, and external references subscales. Post hoc comparisons revealed that the DEP group scored higher on professionals than the HC group, that the OCD group scored higher on self-reassurance than both the DEP and HC groups, and that the DEP group scored higher on the external reference subscale than the OCD group.

Additionally, the three groups differed on the trust subscales. The MANOVA revealed significant main effects of group (between factors), $F(8,122)=2.96, p=.005, p \eta^{2}=.162$. Follow-up ANOVAs revealed significant main effects of group on the health professionals subscale. Post hoc comparisons revealed that the DEP group scored higher on trust in health professionals than the $\mathrm{HC}$ group. 
Table 3. Descriptive statistics for group differences in emotional changes

\begin{tabular}{|c|c|c|c|c|c|c|c|}
\hline \multirow[b]{2}{*}{ Emotion } & \multirow{2}{*}{$\begin{array}{l}\text { Group } \\
\text { Situation }\end{array}$} & \multicolumn{2}{|c|}{ OCD } & \multicolumn{2}{|c|}{ Depression } & \multicolumn{2}{|c|}{ Healthy comparisons } \\
\hline & & Mean & $(S D)$ & Mean & $(S D)$ & Mean & $(S D)$ \\
\hline \multirow[t]{3}{*}{ Reassured } & No reassurance & -3.16 & $(2.490)$ & -2.59 & $(2.265)$ & -1.67 & $(1.465)$ \\
\hline & Short-term & 2.59 & (1.775) & 3.24 & (1.348) & 3.00 & (1.383) \\
\hline & Long-term & 1.00 & $(2.553)$ & 1.88 & $(1.453)$ & 2.54 & $(1.587)$ \\
\hline \multirow[t]{3}{*}{ Anxiety } & No reassurance & 2.81 & $(2.845)$ & 2.24 & (3.093) & 0.63 & $(2.163)$ \\
\hline & Short-term & -1.28 & $(2.727)$ & -1.12 & $(2.315)$ & -1.71 & (1.899) \\
\hline & Long-term & -0.03 & $(2.857)$ & -0.12 & (2.369) & -1.54 & (1.956) \\
\hline \multirow[t]{3}{*}{ Urge to seek more } & No reassurance & 3.22 & $(2.210)$ & -0.06 & (3.897) & 0.22 & (1.953) \\
\hline & Short-term & 0.88 & (2.709) & -0.59 & (1.970) & -1.22 & $(2.173)$ \\
\hline & Long-term & 1.28 & $(2.667)$ & 0.59 & $(2.152)$ & -0.87 & (1.842) \\
\hline \multirow[t]{3}{*}{ Guilt } & No reassurance & 1.31 & $(2.633)$ & 1.29 & (3.601) & 0.39 & $(1.901)$ \\
\hline & Short-term & 1.78 & (2.196) & 0.70 & $(1.490)$ & -0.47 & (1.675) \\
\hline & Long-term & 1.25 & $(2.200)$ & 0.64 & $(1.222)$ & -0.52 & $(1.473)$ \\
\hline
\end{tabular}

Note: $\mathrm{OCD}=$ Obsessive-Compulsive Disorder

The three groups also differed on the intensity subscales. A MANOVA revealed significant main effects of group, $F(8,116)=5.51, p<.001, p \eta^{2}=.275$. Follow-up ANOVAs revealed significant main effects of group on direct seeking of reassurance from people and on selfreassurance. Post hoc comparisons revealed that the OCD group scored significantly higher on direct seeking of reassurance from people, relative to both DEP and HC groups. The OCD group also scored higher on self-reassurance than the HC group.

The three groups also differed on the carefulness subscales. A MANOVA indicated a marginally significant main effect of group, $F(6,128)=1.93, p=.081, p \eta^{2}=.083$. Followup ANOVAs revealed significant main effects of group on the becoming critical and careful listening subscales. Post hoc comparisons revealed that the OCD group scored higher on becoming critical than the HC group, and the DEP group scored higher on careful listening than the HC group.

\section{Group comparison of emotional changes}

Repeated-measures ANOVAs were conducted for changes in feelings of reassurance, anxiety, guilt, and the urge to seek reassurance. These analyses revealed significant effects of group (the between-subjects factor) in changes in feelings of reassurance, $F(2,70)=24.09, p<.001$, $p \eta^{2}=.139$, changes in feelings of anxiety, $F(2,70)=5.24, p<.001, p \eta^{2}=.130$, and changes in feelings of guilt, $F(2,70)=7.893, p=.001, p \eta^{2}=.186$. Post hoc multiple comparisons using Tukey's HSD test revealed that the OCD group had higher ratings than the HC group on these three emotions.

Moreover, repeated-measures ANOVAs revealed significant situation effects (the withingroup factor) in changes in feelings of reassurance, $F(2,70)=175.58, p<.001, p \eta^{2}=.715$, and in changes in feelings of anxiety, $F(2,70)=35.59, p<.001, p \eta^{2}=.337$. Within-factor Bonferroni comparisons revealed that participants rated the feeling of reassurance as greater and the feeling of anxiety as smaller in the short term, than scores for the long term or more 


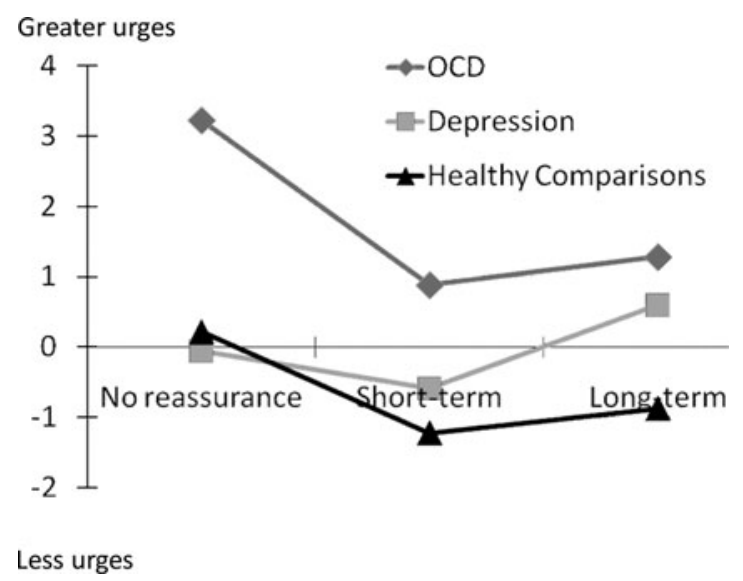

Figure 1. Between-group change in the urge to seek more reassurance for obsessive-compulsive disorder (OCD), depression, and comparison groups

assurance. Further, they rated the feeling of reassurance greater and the feeling of anxiety smaller in the long term than situations in which reassurance was not given. These results suggest that participants report better feelings even in the long term, compared to not obtaining reassurance.

For changes in the urge to seek reassurance, a repeated-measures ANOVA revealed significant effects of group, $F(2,70)=14.01, p<.001, p \eta^{2}=.289$, and situation, $F(2,70)=7.98, p=.001, p \eta^{2}=.104$. The interaction effect was marginally significant, $F(4,68)=2.29, p=.062, p \eta^{2}=.062$ (Figure 1 ). The post hoc analysis of simple main effects for the between-group factor revealed that the OCD group had higher ratings for the no-reassurance situation than the DEP and HC groups; the OCD group also had higher ratings for the short-term situation than the HC group. The post hoc analysis of simple main effects for within-group factors revealed that only the OCD group had a higher rating for the noreassurance situation than for the short-term or long-term situations (Figure 1).

\section{Discussion}

The difference in self-reassurance suggests that individuals with OCD would seek reassurance by telling themselves that there is nothing to worry about, and by checking their memory and notes. In general, individuals in clinical populations would seek reassurance more carefully, making greater effort when seeking reassurance by examining the person carefully, listening carefully, asking others to repeat their statements, and rephrasing the reassurance in their minds in order to examine the "validity" of the reassurance. This aspect of reassurance seeking (in addition to its repetitive nature) may irritate others, and thus elicit negative feedback (Coyne, 1976).

The present study also examined how participants would feel when they received or failed to receive reassurance. Our results suggest that reassurance tends to improve individuals' emotional state by increasing feelings of reassurance and by reducing anxiety and the urge 
to seek more reassurance. This supports the notion that reassurance seeking and compulsive checking are functionally similar (van den Hout and Kindt, 2004; Rachman, 2002; Rachman and Hodgson, 1980; Salkovskis, 1999). The results also make it clear that although individuals with OCD may be aware that the effect of reassurance is relatively transient, their prediction of a diminished long-term positive effect still represents a significant benefit relative to their expectation of what would have happened had they not been reassured. Furthermore, the results indicate that individuals with OCD reported feeling a greater urge to seek reassurance when such reassurance was not provided, whereas such urges to seek reassurance did not increase in individuals with depression. This does not support Coyne's interactional model of depression (Coyne, 1976) possibly because individuals with OCD tend to seek reassurance about perceived threats toward and responsibility for themselves and those close to them (Salkovskis, 1999). Another explanation for our findings is that individuals with depression may try to suppress the urge to seek reassurance because the reassurance provider is often the source of perceived threat (Parrish and Radomsky, 2010).

The present study has several clinical implications in terms of the treatment for OCD. First, clinicians should identify and address self-reassurance in OCD, such as setting up behavioural experiments to test how self-reassurance affects their anxiety in the short- and long term. This is because individuals with OCD may develop self-reassurance as a contingency plan (Kobori et al., 2012), generally considered to be a good strategy from the sufferer's perspective. Second, carefulness of reassurance seeking (as well as its frequency and intensity) should be addressed in treatment because making a greater effort to validate the provided reassurance would be counterproductive and may cause interpersonal difficulties between sufferers and carers (Kobori et al. 2012). Finally, clinicians should acknowledge that for people suffering from OCD, seeking reassurance could be the only possible response in the absence of any helpful alternative. Moreover, carers may provide reassurance even if they believe that reassurance does not solve the problem, because it is still better to offer reassurance than not.

\section{Limitations and future directions}

The present research has several limitations, and many important questions remain open to empirical investigation. First, the sample size was relatively small and limited to only Japanese participants, and participants with depression were not diagnosed using the SCIDIV. Future replication in a larger sample that includes other ethnic/cultural groups with a formal diagnostic procedure will be required to confirm our results. Second, our study did not exclude individuals with OCD who were also depressed. Although it is common in realworld practice to encounter high comorbidity rates among clinical populations, the absence of "pure" OCD and depression groups limited our ability to draw definitive conclusions about the specific effects of depression vs. anxiety on participants' reassurance-seeking behaviours. In particular, because the OCI emphasizes the measurement of compulsions with only one obsessions subscale, it is possible that some participants in the DEP group met the criteria for OCD, although we screened out those who scored above 60. Accordingly, we recommend that future investigations in this area recruit and compare more well-defined clinical groups (where possible).

Because reassurance seeking involves interpersonal processes, it is essential to consider the experiences of others (e.g. partners and family members). Currently, we are investigating how often carers are asked for reassurance, how they are asked (e.g. directly, indirectly, 
asked to take part in rituals), how often they provide reassurance, how they feel and how they think sufferers would feel when they provide or do not provide reassurance, and what motivates them to provide reassurance. So far, no study has experimentally tested the function of reassurance in OCD and depression; for example, as suggested by the spontaneous decay of compulsive urges in OCD, we need to test whether the urge to seek reassurance would increase immediately after reassurance is denied, but subsequently decrease over time (Rachman, de Silva and Roper, 1976; de Silva, Menzies and Shafran, 2003). Moreover, it would be interesting to experimentally investigate what kind of reassurance (e.g. verbal response from others/professionals, self-reassurance, external reference, or assisting rituals) is helpful vs. harmful, and in which circumstances.

\section{Conclusion}

This study is the first to quantitatively elucidate differences in reassurance seeking between OCD and depression. Our results suggest that individuals with OCD seek reassurance more intensely and employ self-reassurance more frequently than do healthy individuals or individuals with depression. In addition, compared to healthy individuals or individuals with depression, individuals with OCD appear to feel a greater need to seek more reassurance when reassurance is not given.

\section{Acknowledgements}

This work was supported by Grants-in-Aid for Young Scientists (B) from the Japan Society for Promotion of Science.

\section{Ethical standards}

The authors assert that all procedures contributing to this work comply with the ethical standards of the relevant national and institutional committees on human experimentation and with the Helsinki Declaration of 1975, as revised in 2008. Additionally, the study was approved by the Research Ethics Committee at Chiba University.

\section{References}

Beck, A. T., Steer, R. A. and Brown, G. K. (1996). Manual for the Beck Depression Inventory-2. San Antonio, TX: Psychological Corporation.

Brislin, R. W. (1970). Back-translation for cross-cultural research. Journal of Cross-Cultural Psychology, 1, 185-216.

Brislin, R. W. (1986). The wording and translation of research instruments. In W.J. Lonner and J.W. Berry (Eds.), Field Methods in Cross-Cultural Research (pp. 137-164). Thousand Oaks, CA: Sage.

Coyne, J. C. (1976). Toward an interactional description of depression. Psychiatry, 39, 28-40.

de Silva, P., Menzies, R. G. and Shafran, R. (2003). Spontaneous decay of compulsive urges: the case of covert compulsions. Behaviour Research and Therapy, 41, 129-137. 
First, M., Spitzer, R., Gibbon, M. and Williams, J. (1995). Structured Clinical Interview for DSM-IV Axis I Disorders-Patient edition. New York: Biometrics Research Department, NY State Psychiatric Institute.

Foa, E. B., Kozak, M. J., Salkovskis, P. M., Coles, M. E. and Amir, N. (1998). The validation of a new obsessive-compulsive disorder scale: the Obsessive-Compulsive Inventory. Psychological Assessment, 3, 206-214.

Ishikawa, R., Kobori, O. and Shimizu, E. (2013). Development and validation of the Japanese version of the Obsessive-Compulsive Inventory. Manuscript submitted for publication.

Joiner, T. E., Metalsky, G. I., Katz, J. and Beach, S. R. H. (1999). Depression and excessive reassurance seeking. Psychological Inquiry, 10, 269-278.

Kobori, O. and Salkovskis, P. M. (2013). Patterns of reassurance seeking and reassurance-related behaviours in OCD and anxiety disorders. Behavioural and Cognitive Psychotherapy, 41, 1-23.

Kobori, O., Salkovskis, P. M., Read, J., Lounes, N. and Wong, V. (2012). A qualitative study of the investigation of reassurance seeking in obsessive-compulsive disorder. Journal of ObsessiveCompulsive and Related Disorders, 1, 25-32

Kobori, O. and Sawamiya, Y. (2012). Development of the Japanese version of Reassurance Seeking Questionnaire. Unpublished manuscript.

Kojima, M., Furukawa, T. A., Takahashi, H., Kawai, M., Nagaya, T. and Tokudome, S. (2002). Cross-cultural validation of the Beck Depression Inventory-II in Japan. Psychiatry Research, 110, 291-299.

Parrish, C. L. and Radomsky, A. S. (2006). An experimental investigation of responsibility and reassurance: relationships with compulsive checking. International Journal of Behavioural and Consultation Therapy, 2, 174-191.

Parrish, C. L. and Radomsky, A. S. (2010). Why do people seek reassurance and check repeatedly? An investigation of factors involved in compulsive behaviour in OCD and depression. Journal of Anxiety Disorders, 24, 211-222.

Rachman, S. J. (2002). A cognitive theory of compulsive checking. Behaviour Research and Therapy, 40, 625-639.

Rachman, S. J., de Silva, P. and Roper, G. (1976). Spontaneous decay of compulsive urges. Behaviour Research and Therapy, 14, 445-453.

Rachman, S. J. and Hodgson, R. J. (1980). Obsessions and Compulsions. Englewood Cliffs, NJ: Prentice Hall.

Salkovskis, P. M. (1999). Understanding and treating obsessive-compulsive disorder. Behaviour Research and Therapy, 37, S29-S52.

Salkovskis, P. M. and Kobori, O. (2013). Reassuringly calm? Self-reported patterns of responses to reassurance seeking in Obsessive Compulsive Disorder. Manuscript submitted for publication.

Starr, L. R. and Davila, J. (2008). Excessive reassurance seeking, depression, and inter-personal rejection: a meta-analytic review. Journal of Abnormal Psychology, 117, 762-775.

van den Hout, M. and Kindt, M. (2004). Obsessive-compulsive disorder and the paradoxical effects of perseverative behaviour on experienced uncertainty. Journal of Behaviour Therapy and Experimental Psychiatry, 35, 165-181. 